\title{
Is Preoperative Functional Status Associated with Postoperative Mortality and Morbidity in Elective Open Heart Patients?
}

\author{
A. Kate MacPhedran ${ }^{1,2}$, David B. Barker ${ }^{3}$, Mark L. Marbey ${ }^{2,4}$, Kieran Fogarty $^{5}$, Eric Vangsnes ${ }^{6}$ \\ ${ }^{1}$ Doctor of Physical Therapy Program Department, Gannon University, Erie, USA \\ ${ }^{2}$ Saint Vincent Health Center, Erie, USA \\ ${ }^{3}$ Organizational Learning and Leadership Ph.D. Program, Gannon University, Erie, USA \\ ${ }^{4}$ Mason City Clinic, Mason City, USA \\ ${ }^{5}$ Interdisciplinary Health Sciences Ph.D. Program, Western Michigan University, Kalamazoo, USA \\ ${ }^{6}$ Physician Assistant Program, Western Michigan University, Kalamazoo, USA \\ Email:*macphedr001@gannon.edu
}

How to cite this paper: MacPhedran, A.K., Barker, D.B., Marbey, M.L., Fogarty, K. and Vangsnes, E. (2018) Is Preoperative Functional Status Associated with Postoperative Mortality and Morbidity in Elective Open Heart Patients? Health, 10, 654-666. https://doi.org/10.4236/health.2018.105051

Received: April 4, 2018

Accepted: May 25, 2018

Published: May 28, 2018

Copyright ( 92018 by authors and Scientific Research Publishing Inc. This work is licensed under the Creative Commons Attribution International License (CC BY 4.0).

http://creativecommons.org/licenses/by/4.0/

c) (i) Open Access

\begin{abstract}
Background: Cardiac surgery, even when planned, has the potential for adverse outcomes, such that several factors are taken into consideration to help surgeons and their patients discuss the potential risks weighed against the expected recovery. Preoperative functional status and its influence on cardiac surgery, if any, have not been adequately evaluated to date. This study aimed to examine the relationship between preoperative functional status and postoperative mortality and morbidity in elective open heart patients. Methods: Preoperative baseline data ( $n=43$ subjects) were obtained to calculate Society of Thoracic Surgeons (STS) mortality and morbidity risk scores and preoperative functional status was measured using the Late-Life Function and Disability Instrument (LLFDI). Follow-up data were abstracted at one year postoperative to calculate actual mortality and morbidity events. Ordinary least squares and negative binomial regression analyses were conducted to assess the relationship between the LLFDI preoperative score to the STS mortality and morbidity risk scores. Results: Mortality risk was significant, $\mathrm{F}(1,39)=$ $4.75, \mathrm{p}=0.035$, with an adjusted $\mathrm{R} 2=0.086$, and Function Total (measured by LLFDI) yielded a significant negative association with mortality risk, $\beta=$ -0.329 . Morbidity was found to be significant, $F(1,40)=4.89, \mathrm{p}=0.033$, with an adjusted R2 $=0.087$ and Function Total yielded a significant negative association with morbidity risk, $\beta=-0.328$, as well. Estimation of the counts for postoperative complications as estimated by Function Total failed to reach significance (Wald $\chi^{2}=0.34, \mathrm{p}=0.56$ ), which provided a pseudo $\mathrm{R} 2=0.009$.
\end{abstract}


Probabilities for frequencies of adverse events (major complications), therefore, could not be reliably calculated. Conclusion: Preoperative diminished functional status, as measured by the LLFDI, is associated with an increased risk of mortality and morbidity in patients undergoing elective cardiac surgery. The risks and benefits of cardiac surgery should be weighed carefully and include a patient's preoperative functional status, especially in the case of an elective procedure.

\section{Keywords}

Functional Status, Elective Cardiac Surgery, Mortality and Morbidity

\section{Introduction}

Coronary artery disease is the leading cause of death worldwide [1]. As patients continue to live longer, the decision becomes less clear on whether the benefits outweigh the risks of undergoing coronary bypass surgery. Specific risk factors (e.g., unstable angina, recent myocardial infarction) as well as medical history (e.g., diabetes, hypertension, prior cardiac surgery) are considered when surgeons estimate the likelihood of complications (morbidity)or mortality after coronary bypass or valve surgery [1] [2] [3] [4] [5]. Physiological factors like body mass index and advanced age have more recently been accepted as additional cardiac surgery variables [1] [6] [7] [8], but the impact of preoperative functional status on cardiac surgery, has not been adequately evaluated to date [8] [9] [10] [11] [12]. As part of the cardiac surgery guideline revisions in 2008, patients of advanced age (at least 70 years old) accounted for $50 \%$ of cardiac surgeries performed in North America and 78\% of the combined major complications (morbidities) and deaths (mortalities) [1]. In terms of valve surgery and aortic valve replacement in particular, older adults ( $\geq 70$ years of age) accounted for $30 \%-40 \%$ of the cases turned down for surgical intervention, despite evidence of surgical success in their age group [4]. In this same age bracket, more than $25 \%$ of these older Americans were functionally limited by cardiovascular disease, according to the United States Census Bureau [9]. Heart disease, either coronary or valvular in nature, is typically a silent disease which progresses gradually over time. It is not until changes are seen in endurance, physical mobility and/or socialization (one's quality of life), that the impact of the disease becomes evident. Likewise, changes in functional status are gradual over time and affect not only physical, emotional and mental well-being, but may interfere with the recovery process. Functional status, as defined for use in this study, is the ability to function physically, perform tasks with both upper and lower extremities, to a degree which provides satisfaction in valued areas of life such as activities of daily living, recreational activities, and interpersonal relationships.

In research, "impaired functional status" has become somewhat synonymous with the term "frailty", and its association with mortality and morbidity has been 
studied primarily in the inpatient, non-surgical population. Narain et al. studied older adult inpatients with varied diagnoses and concluded that, more than the admitting diagnosis [13], decreased functional status was the strongest predictor of 6-month mortality, prolonged length of stay, and readmissions to the hospital. Inouye et al. found a strong association between impaired functional status and mortality among older, non-cardiac patients in the hospital setting, which prompted the recommendation for risk adjustment tools to include a functional status variable, especially for older patients [14]. Purser et al. determined that there was a strong association between slow gait speed (a dimension of frailty) using a short walk test (referred to as the 5-m gait speed test), and 6-month mortality in hospitalized patients treated for coronary artery disease, and also recommended adding some frailty component to risk assessments [15]. Cervera et al. were one of the few to study the effect of preoperative functional status on mortality in the coronary artery bypass graft (CABG) population, though the definition of impaired functional status was limited to anyone who used assistance or an assistive device to ambulate, or had equipment needs such as dialysis or oxygen [9]. Interestingly, Cervera et al. did not find limited functional status to be a predictor of early morbidity or mortality with CABG patients, however, this was a veteran only population, composed almost exclusively of males [9]. A large Canadian study by Lee et al. examined patients undergoing elective cardiac surgery and concluded those who had higher mortality rates were predominately the ones considered frail (those who had impaired ambulation or limited daily living activities), however, Lee et al. did not compare their "frailty" sub-group to any cardiac risk score for predictive validity [10].

From a clinical perspective, cardiac surgeons have universally accepted risk score assessment systems [1] [16] [17] such as the EuroSCORE [17] or Parsonnet score [17] to predict mortality and morbidity risk. The Society of Thoracic Surgeons (STS) risk scoring model is the national standard used in the United States [8]. Despite their wide use and acceptability, risk score systems produce only modest mortality predictions, statistically speaking, and perform poorly in predicting morbidity, as they were not originally intended or designed to detect morbidity risk [8] [12] [16] [17]. Cardiac risk models do not take into consideration patients' functional status as part of the risk stratification calculation [1] [8] [17] despite the fact that the American College of Cardiology valve treatment guideline revision in 2012 acknowledged that frailty (a.k.a. "impaired functional status" in research) may be an important outcome predictor in high-risk populations especially [4].

STS made a recommendation in May, 2011, that preoperative functional measures such as gait speed be added to the STS database for adult patients pending cardiac surgery, in order to aid in stratifying risk [8] [18]. This recommendation was based on findings from a multicenter study in the USA and Canada lead by Afilalo et al. which concluded that slow gait speed utilizing a 5-m distance demonstrated a 2 - 3 fold increase in STS-predicted mortality or major morbidity. Going beyond the 5-m walk test, Sundermann et al. found sig- 
nificant associations in their more comprehensive frailty assessment and its predictive validity with respect to early and 1-year mortality as well as morbidity in elective cardiac surgery patients [11] [12]. The comprehensive assessment of frailty (CAF) tool by Sundermann et al., however, is laborious to conduct for clinicians, and as yet, has not shown to be superior to cardiac risk score assessments [11]. There has been limited preoperative functional data collected to date since the STS announcement and no standardized approach taken with this recommendation to evaluate its impact on mortality and morbidity risk [8] [9] [10] [11] [12] [18] [19].

The gold standard for measuring physical function and capacity has been the six-minute walk test but it only accounts for the "physical" component [20] [21]. The Late-Life Function and Disability Instrument (LLFDI) is a self-reported questionnaire specifically targeting a wide variety of physical activities (function) and social life tasks (disability status), which defines one's functional status [22]. This outcome tool was designed to assess community-dwelling and ambulating 75 to 90 year old adults, and its use has been validated on patients with cardiovascular disease and post-cardiac surgery [21] [23] [24] [25] [26]. The LLFDI correlated significantly with the six-minute walk test, but also with the $4.5 \mathrm{~m}$ walk test, Short Physical Performance Battery, Timed Up and Go, and the Short Form 36 (all widely accepted functional measures) in its concurrent validity, reliability, precision, and responsiveness with diverse patient populations [27] [28] [29] [30]. More comprehensive and perhaps more unique than any of the above tests mentioned, the LLFDI also indicates aspects on recreational participation and community socialization which significantly impacts one's quality of life and makes this an appropriate tool to use with the cardiac surgical population.

As of 2010, the risk-adjusted mortality rate for isolated CABG was 2.1\% [2] and 2.6\% for isolated AVR [4] per the 2012 updated guidelines, yet admittedly these models fail to include potentially influential risk factors such as impaired functional status [4]. While the LLFDI has been proven valid and reliable as a tool to measure functional status in a comparable population, to date, there have not been any published studies exploring its predictive validity with respect to mortality and morbidity [30].

\section{Methods}

\subsection{Study Design}

A non-experimental design using a prospective cohort of subjects undergoing elective cardiac surgery from Saint Vincent Health Center between June and December 2010 was assembled. Preoperative baseline data was obtained to calculate mortality and morbidity risk and follow-up postoperative data was abstracted at one year to calculate actual mortality and morbidity events. Regression analysis was conducted to assess the relationship between the LLFDI preoperative score (independent/predictor variable) to the STS mortality and morbidity risk scores (dependent/outcome variables). 


\subsection{Participants}

Subjects included in this study were at least 18 years old, able to communicate fluently in English, and underwent one of the following elective cardiac surgeries: initial or redo coronary artery bypass graft (CABG), valve repair/replacement, or any valve/CABG combination procedure. If elective cardiac surgery became emergency surgery or a subject failed to submit or sufficiently complete their preoperative LLFDI form, they were excluded/terminated from the study. Consecutive subjects were screened after they were scheduled to undergo cardiac surgery which involved CABG, valve repair/replacement, or valve and CABG combination surgery. Eligible subjects were asked to complete a LLFDI questionnaire preoperatively and this served as the predictor variable for this study. This study was approved by the human subjects review committees at Saint Vincent Health Center and Western Michigan University.

\subsection{Measures}

In this study, functional status was measured using only the Functional component of the LLFDI tool. The Disability component focuses more on recreational tasks and community socialization than the daily movements and limitations asked in the Function component [22] [23] [24]. Since both the Function and Disability components each have strong validity and reliability, they can be used as stand-alone tests [21] [22] [23] [24] [29]. The Function component is made up of (32) questions using a 0 to 5 Likert scale that start with asking, "How much difficulty do you have?" on routine physical actions and daily activities such as making a bed or walking up a flight of stairs [22] [23] [24]. The higher the Function score, the more functionally able/active one is. Each question carries a different weight [21] [22] [23] [24] [29], therefore, the raw scores were converted to 0 - 100 scaled scores using an accompanying LLFDI computer program.

\subsubsection{Late-Life Function and Disability Instrument (LLFDI)}

The specific demographic and clinical patient information found in Table 1 has been identified by the STS to collectively constitute the significant mortality and morbidity risk predictors in cardiac surgery [13] [14] [16] [17] [31], and is the same information used to calculate these two outcome variables for this study. The STS cardiac risk score calculator data version 2.81 was developed in 2007 and allows health care workers and researchers to estimate individual mortality and morbidity risk for cardiac surgery by entering individual clinical data points based on these predicted risks [5]. With assistance from the STS data abstractor (someone who locates and receives information from medical records and prepares the data for a requester), preoperative clinical data were derived from chart reviews prospectively to determine the initial mortality risk, which was calculated using the STS risk calculator version 2.81. The STS-mortality risk (in percentage) is based on 24 covariates [4] ("Preoperative data" column in Table 1), individually weighed, and involves mathematical formulas for deriving the end calculations which are all proprietary information of STS [5]. The STS risk 
Table 1. Data abstracted for sts risk estimates and actual postoperative events.

\begin{tabular}{cc}
\hline Preoperative & Postoperative \\
\hline Age & Specific cardiac surgical procedure \\
Gender & Hospital length of stay \\
Ethnicity & Postoperative bleeding (return to O.R.; blood products) \\
Ejection fraction \% (EF) & Deep sternal infection \\
NYHA Classification & Intubation > 24 hours (and re-intubation) \\
Creatinine level & Creatinine within 72 hours \\
Prior cardiovascular surgery & Adverse arrhythmia (pacemaker/defibrillator required) \\
Number of vessel disease & Total cross-clamp time \\
Myocardial Infarct history & Mortality (all-cause, at 30-days \& 1-year) \\
Prior neurologic event & Neurologic event \\
Co-morbidities & Co-morbidities (progressed/new diagnoses) \\
Valve disease/insufficiency & \\
Body Mass Index (BMI) &
\end{tabular}

calculator version 2.81 also provides a combined "mortality or morbidity risk" estimate (in percentage) from the same calculation method used to estimate mortality risk. For this study, the morbidity risk was calculated by subtracting the estimated "mortality risk" score from the "mortality or morbidity risk" score, since STS does not directly estimate morbidity on its own.

\subsubsection{STS Risk Calculator}

Actual adverse events, which comprise the basis for morbidity risk according to the STS, is a composite based on any of the following 5 major complications (found in "Postoperative Data" in Table 1): stroke (permanent neurologic event $>24$ hours as confirmed by diagnostic testing), renal failure (new requirement for dialysis or creatinine level at 3 -fold increase from preoperative level), prolonged ventilation ( $>24$ hours or reintubation required), deep sternal wound infection (requiring operative intervention and antibiotic therapy with positive cultures), and need for reoperation (due to major bleeding), as well as postoperative death (all-cause by 30 days and by one year-postoperative) [1] [5] [8]. The frequency of major complications (a.k.a. adverse events, bolded in Table 1 under "Postoperative Data") were calculated in this study and compared against the individual's predicted morbidity risk score as estimated by the LLFDI.

Because mortality and morbidity data is often extended beyond the patients' hospitalization, including postoperative data at 30 days and as far out as one year [16] [31], retrospective data abstraction was also used to capture this trend. The additional postoperative clinical data (entire "Postoperative Data" column in Table 1) were abstracted prospectively from chart reviews during the subject's hospitalization and again retrospectively one year post-surgery, in order to cal- 
culate actual complications (morbidity events stated above), any readmissions within 30 days, as well as any listing of death (checked in medical records and obituary searches statewide). Data from the subjects' medical records (Table 1) were accessed and extracted using McKesson Electronic Medical Record Systems and MIS medical records and compiled on a disc. All information was transferred onto an Excel spreadsheet, de-identified, and verified for accuracy against the hardcopy, which was then destroyed.

\subsection{Data Analysis}

All data were analyzed using the statistical package Stata 14 (StataCorp, College Station, TX). Study demographics were collected (Table 2) on gender, age, and race/ethnicity as well as preoperative functional status (measured by the LLFDI Function Total score), and surgical mortality and morbidity risk as based on the STS risk calculator scores (in percentage). Descriptive statistics on the sample demographics were examined for frequency distribution and assumptions of normality. Ordinary least square regression was conducted to estimate mortality and morbidity risk using preoperative functional status (LLFDI Function Total) as the explanatory variable. Negative binomial regression was conducted to estimate the frequency and probability of adverse events (major complications) utilizing the LLFDI. Data were considered significant at $\mathrm{p}<0.05$.

\section{Results}

\subsection{Baseline Demographics and Variable Distribution}

The study cohort consisted of 43 subjects with completed preoperative LLFDIs for analysis (Figure 1). Subjects ranged from 45 to 83 years with a mean age of $66 \pm 9.7$ years; 28\% were female and 95\% were Caucasian (Table 2). All 43 subjects were alive 30 days postoperative; however, one (2.3\%) subject had died within one year post-surgery. Eighty-four percent of the cohort underwent coronary bypass graft surgery (initial or redo), but for analysis purposes, all cardiac surgical procedures were combined, including valve surgeries, which made up $16 \%$ (repair, replacement, or in combination with bypass surgery).

Functional status, mortality and morbidity risk scores were assessed for normality, however; both mortality and morbidity risk scores evidenced significant departure from normality when Shapiro-Wilk test was conducted. Quantile-normal plots were generated to determine best fit for transformation and log transformation was determined for both outcome variables. After the necessary variables were transformed, all the assumptions of normality were met for analysis purposes.

\subsection{Regression Analysis}

The regression of mortality risk on functional status was found to be significant, $\mathrm{F}(1,41)=4.96, \mathrm{p}=0.032$, providing an adjusted $\mathrm{R}^{2}=0.086$. Function Total yielded a significant negative association with mortality risk, $\beta=-0.328$. 
Table 2. Patient characteristics and LLFDI validation demographics.

\begin{tabular}{|c|c|c|}
\hline & $\begin{array}{l}\text { Patient Characteristics } \\
\quad \text { (based on } n=43 \text { ) }\end{array}$ & $\begin{array}{l}\text { LLFDI demographics } \\
\quad(\text { based on } \mathrm{n}=150)\end{array}$ \\
\hline \multirow{2}{*}{ Gender } & $12(28 \%)$ & Female $23 \%$ \\
\hline & $31(72 \%)$ & Male $\mathbf{7 7 \%}$ \\
\hline \multirow[t]{2}{*}{ Age } & $\begin{array}{l}\text { Range } 45-83 \text { years } \\
\text { (SD 9.74) } \mathbf{M}=66.35^{*}\end{array}$ & Range 60 - 90 years \\
\hline & Caucasian $41(95 \%)$ & $84 \%$ \\
\hline \multirow[t]{4}{*}{ Race/Ethnicity } & Hispanic or Latino $1(2 \%)$ & $5 \%$ \\
\hline & African American 1 (2\%) & $7 \%$ \\
\hline & CABG—on pump $30(72.1 \%)$ & --- \\
\hline & CABG- off pump $5(11.6 \%)$ & --- \\
\hline \multirow[t]{2}{*}{ Surgical Approach } & CABG - both types combined, 35 (83.7\%) & --- \\
\hline & $\begin{array}{l}\text { Valve replacement/repair alone or with } \\
\qquad \text { CABG } 8(16.3 \%)\end{array}$ & --- \\
\hline $\begin{array}{c}\text { Preoperative } \\
\text { Functional Status }\end{array}$ & $\begin{array}{l}\text { LLFDI Function Total } \\
\text { (score range } 0-100) \\
\mathrm{M}=\mathbf{6 1 . 3 9}(\mathrm{SD} 9.41)\end{array}$ & $\begin{array}{l}\text { LLFDI Function Total } \\
\text { (score range } 0-100) \\
M=62.9(S D 13.0)\end{array}$ \\
\hline Mortality Risk (\%) & $\mathrm{M}=1.47(\mathrm{SD} 1.31)$ & $\mathrm{n} / \mathrm{t}$ \\
\hline Morbidity Risk (\%) & $\mathrm{M}=10.23(\mathrm{SD} 4.27)$ & $\mathrm{n} / \mathrm{t}$ \\
\hline
\end{tabular}

Late-Life Function and Disability Instrumentn/t = not tested. CABG = Coronary Artery Bypass Graft *compared to national mean age of 64.9 years for CABG (STS, 2010).

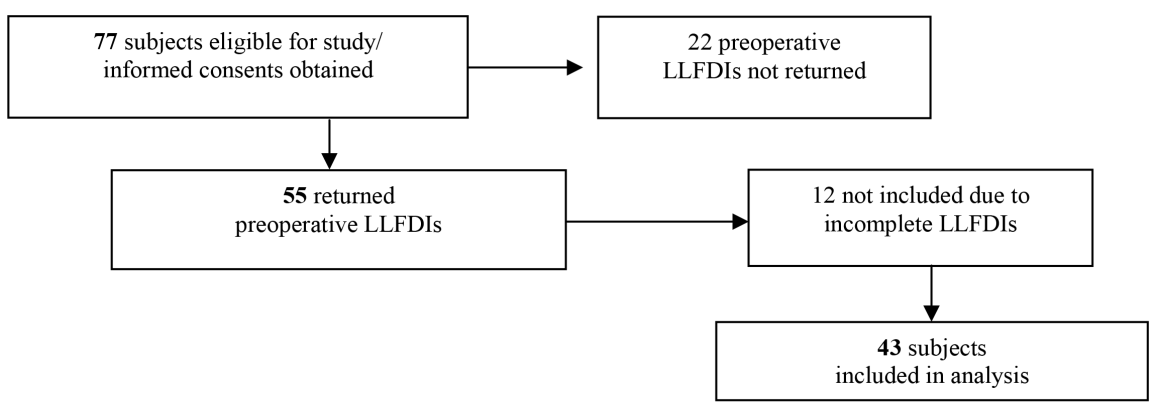

Figure 1. Late-Life Function and Disability Instrument (LLFDI) data flow diagram.

Regression diagnostics indicated that normality of residuals and homoscedasticity assumptions were met. Analysis to detect influential observations using Cook's distance revealed the presence of two potentially influential cases. Both cases were deleted and the analysis was rerun. The equation for mortality (Table 3) was found to be significant, $F(1,39)=4.75, p=0.035$, with an adjusted $R^{2}=$ 0.086 , and Function Total yielded a significant negative association with mortality risk, $\beta=-0.329$.

The equation for morbidity risk was not significant, $\mathrm{F}(1,41)=2.66, \mathrm{p}=0.11$, providing an adjusted $\mathrm{R}^{2}=0.038$. Function Total yielded a significant negative association with morbidity risk, $\beta=-0.247$. Normality of residuals and homoscedasticity assumptions were met. Analysis for the presence of influential 
Table 3. Bivariate least squares regression analysis: mortality risk $(N=41)$.

\begin{tabular}{ccccc}
\hline Variable & $B$ & SE B & $\beta$ & Adjusted $\mathrm{R}^{2 \neq}$ \\
\hline Functional Total & -0.044 & 0.013 & $-0.469^{*}$ & 0.086 \\
\hline${ }^{*} \mathrm{p}<0.05 ;{ }^{* *} \mathrm{p}<0.01 ;{ }^{\ddagger}$ Adjusted $\mathrm{R}^{2}$ was based on reverse transformation of the dependent variable; $B$ \\
$=$ Slope; $\mathrm{SE} \mathrm{B}=$ Standard Error of Slope; $\beta=$ Standardized Slope.
\end{tabular}

observations using Cook's distance revealed one potentially influential case. The regression equation for morbidity following deletion of this observation (Table 4) was found to be significant, $F(1,40)=4.89, \mathrm{p}=0.033$, with an adjusted $\mathrm{R}^{2}=$ 0.087 and Function Total yielded a significant negative association with morbidity risk, $\beta=-0.328$.

Results for the negative binomial regression analysis appear in Table 5. Estimation of the counts for postoperative complications as estimated by Function Total failed to reach significance (Wald $\chi^{2}=0.34, p=0.56$ ), which provided a pseudo $R^{2}=0.009$. Consequently, probabilities for frequencies of adverse events (major complications) could not be reliably calculated.

\section{Discussion and Conclusion}

Preoperative functional status, as measured by LLFDI Function Total, yielded significant findings in predicting both mortality and morbidity risk in elective cardiac surgery. Although the LLFDI outcome measure has been widely used with the cardiac population (e.g., cardiac rehab post-bypass surgery, coronary heart disease, congestive heart failure) [26] [30] [32], to date, this is the only known research study to have explored the predictive validity of the LLFDI in terms of mortality and morbidity risk in elective cardiac surgery patients [30]. What is not known is how much of the variance for mortality or morbidity risk can be accounted for by LLFDI preoperative Function Total. With such a small sample size, comparing the LLFDI is infeasible against such an exhaustive list of cardiac surgery risk factors that includes every possible influencing variable [1] [8] [9]. A larger sample in the future would help detect if clinically relevant effects occurred and may strengthen the overall power of this study.

Despite the overall significance found with the LLFDI, there were two cases deemed the exception as they were flagged as unduly influencing the mortality risk results due to their individual functional scores: one was unexpectedly high and the other was extremely low. Consequently, the LLFDI was a poor predictor of mortality risk in these two cases. As stated earlier, there are numerous potential variables that can influence cardiac surgery complications and mortality. It is essential to isolate as many of the key influencing variables as possible when using surgical risk predictor tools such as STS, otherwise calculating the estimate may be flawed, and on elective procedures especially, accuracy is paramount. In an attempt to examine the effect of the LLFDI alone, there certainly could have been another unexplained covariate(s) acting or anomalies occurring within the accounted STS variables. 
Table 4. Bivariate least squares regression analysis: morbidity risk $(N=42)$.

\begin{tabular}{ccccc}
\hline Variable & $B$ & SE B & $\beta$ & Adjusted $\mathrm{R}^{2 \neq}$ \\
\hline Functional Total & -0.014 & 0.006 & $-\mathbf{0 . 3 2 8}^{*}$ & 0.087 \\
\hline${ }^{*} \mathrm{p}<0.05 ;{ }^{* *} \mathrm{p}<0.01 ;{ }^{*}$ Adjusted $\mathrm{R}^{2}$ was based on reverse transformation of the dependent variable; $B$ \\
$=$ Slope; $\mathrm{SE} \mathrm{B}=$ Standard Error of Slope; $\beta=$ Standardized Slope.
\end{tabular}

Table 5. Negative binomial regression analysis: Frequency of complications $(\mathrm{N}=43)$.

\begin{tabular}{cccc}
\hline Variable & $B$ & SE B & Pseudo R $^{2}$ \\
\hline Functional Total & -0.031 & 0.053 & 0.009 \\
\hline${ }^{*} \mathrm{p}<0.05 ;{ }^{* *} \mathrm{p}<0.01 ; B=$ Slope; SE B $=$ Standard Error of Slope. &
\end{tabular}

The LLFDI overestimated morbidity in one case and consequently was a poor predictor of morbidity risk in this one case. Interestingly, this case was one of the two cases that was also problematic with regard to mortality risk. Overall, results of the regression analysis were an accurate reflection of the association between the variables in the vast majority of the cases. Furthermore, finding a significant association between the LLFDI Function Total and STS morbidity risk, as well as LLFDI Function Total and STS mortality risk, suggests that the STS cardiac risk score may need to be refined.

This study had limitations with attrition rate from obtaining consents to receiving preoperative LLFDIs of $29 \%(\mathrm{n}=22)$. One explanation may have been that subjects were met briefly and typically after they just received news of needing cardiac surgery without much time to process all of the information. Unfortunately, there is only a small window of time (roughly two hours) to approach these potential subjects in person after all of their consults and tests have been completed and before they are discharged. This issue could potentially be improved in future studies by conducting a follow-up call at home 1 - 2 days after the initial contact and prior to surgery.

Conducting this study at a single site with a sample of convenience (i.e., elective, primarily on-pump cases) without a control group certainly challenges the ability to generalize findings to the cardiac surgery population. Closer examination of the data revealed that all but five of the participating subjects that underwent CABG surgery $(n=35)$, were performed on-pump, and of the eight subjects who underwent valve repair or replacement, three of the valve procedures was a combination valve/CABG surgery. Additionally, two of the valve cases were mitral valve surgeries, which naturally carry higher mortality risk, however; all of these sub-groups were too small to do any comparative studies on mortality and morbidity influence. Regardless of the cardiac surgery performed, however, preoperative diminished functional status, as measured by the Late-Life Function and Disability Instrument, is associated with an increased risk of mortality and morbidity in patients undergoing elective cardiac surgery. The risks and benefits of cardiac surgery should be weighed carefully and in- 
clude a patient's preoperative functional status, especially in the case of an elective procedure.

\section{Acknowledgements}

The researchers involved in this study would like to thank all the participants who took part in this study and their families who assisted them along the way.

\section{Conflicts of Interests}

There were no conflicts of interests to disclose for any of the authors. This study was not funded by any grant or supported by any external source.

\section{References}

[1] Shahian, D.M., O’Brien, S.M., Filardo, G., Ferraris, V.A., Haan, C.K., Rich, J.B., Anderson, R.P., et al. (2009) The Society of Thoracic Surgeons 2008 Cardiac Surgery risk Models: Introduction and Part 1 -Coronary Artery Bypass Grafting Surgery. Annals of Thoracic Surgery, 88, S1-S22. https://doi.org/10.1016/j.athoracsur.2009.05.053

[2] Hillis, L., Smith, P.K., Anderson, J.L., Bittl, J.A., Bridges, C.R., Byrne, J.G., Winniford, M.D., et al. (2011) 2011 ACCF/AHA Guideline for Coronary Artery Bypass Graft Surgery: Executive Summary. A Report of the American College of Cardiology Foundation/American Heart Association Task Force on Practice Guidelines Developed in Collaboration with the American Association for Thoracic Surgery, Society of Cardiovascular Anesthesiologists, and Society of Thoracic Surgeons. Journal of American College of Cardiology, 58, 2584-2614.

https://doi.org/10.1016/j.jacc.2011.08.008

[3] Roger, V.L., Go, A.S., Lloyd-Jones, D.M., Adams, R.J., Berry, J.D., Brown, T.M., Wylie-Rosett, J., et al. (2011) American Heart Association. Heart Disease and Stroke Statistics 2011 Update: A Report from the American Heart Association. Circulation, 123, e18-e209. https://doi.org/10.1161/CIR.0b013e3182009701

[4] Holmes, D.R., Mack, M.J., Kaul, S., Agnihotri, A., Alexander, K.P., Bailey, S.R., Thomas, J.D., et al. (2012) ACCF/AATS/SCAI/STS Expert Consensus Document on Transcatheter Aortic Valve Replacement. Journal of American College of Cardiology, 59, 1200-1254. https://doi.org/10.1016/j.jacc.2012.01.001

[5] Society of Thoracic Surgeons (2015) STS Adult Cardiac Surgery Database. About the STS Risk Calculator (Version 2.81). Updated 2015. http://riskcalc.sts.org/stswebriskcalc/\#/support.

[6] van Straten, A.H.M., Bramer, S., Soliman Hamad, M.A., van Zundert, A.A.J., Martens, E.J., Schönberger, J.P.A.M. and de Wolf, A.M. (2010) Effect of Body Mass Index on Early and Late Mortality after Coronary Artery Bypass Grafting. Annals of Thoracic Surgery, 89, 30-37. https://doi.org/10.1016/j.athoracsur.2009.09.050

[7] Bakaeen, F.G., Chu, D., Huh, J. and Carabello, B.A. (2010) Is an Age of 80 Years or Greater an Important Predictor of Short-Term Outcomes of Isolated Aortic Valve Replacement in Veterans? Annals of Thoracic Surgery, 90, 769-774. https://doi.org/10.1016/j.athoracsur.2010.04.066

[8] Afilalo, J., Eisenberg, M.J., Morin, J.F., Bergman, H., Monette, J., Noiseux, N., Boivin, J-F., et al. (2010) Gait Speed as an Incremental Predictor of Mortality and Major Morbidity in Elderly Patients Undergoing Cardiac Surgery. Journal of American College of Cardiology, 56, 1668-1676. https://doi.org/10.1016/j.jacc.2010.06.039 
[9] Cervera, R., Bakaeen, F.G., Cornwell, L.D., Wang, X.L., Coselli, J.S., Le Maire, S.A. and Chu, D. (2012) Impact of Functional Status on Survival after Coronary Artery Bypass Grafting in a Veteran Population. Annals of Thoracic Surgery, 93, 1950-1955. https://doi.org/10.1016/j.athoracsur.2012.02.071

[10] Lee, D.H., Buth, K.J., Martin, B.J., Yip, A.M. and Hirsch, G.M. (2010) Frail Patients Are at Increased Risk for Mortality and Prolonged Institutional Care after Cardiac Surgery. Circulation, 121, 973-978. https://doi.org/10.1161/CIRCULATIONAHA.108.841437

[11] Sündermann, S.H., Dademasch, A., Praetorius, J., Kempfert, J., Dewey, T., Folk, V., Walther, T., et al. (2011) Comprehensive Assessment of Frailty for Elderly High-Risk Patients Undergoing Cardiac Surgery. European Journal of Cardio-Thoracic Surgery, 39, 33-37. https://doi.org/10.1016/j.ejcts.2010.04.013

[12] Sündermann, S.H., Dademasch, A., Seifert, B., Biefer, H.R.C., Emmert, M.Y., Walther, T., Starck, C.T., et al. (2014) Frailty Is a Predictor of Short- and Mid-Term Mortality after Elective Cardiac Surgery Independently of Age. Interactive Cardiovascular and Thoracic Surgery, 18, 580-585. https://doi.org/10.1093/icvts/ivu006

[13] Narain, P., Rubenstein, L.Z., Wieland, G.D., Rosbrook, B., Strome, L.S., Pietruszka, F. and Morley, J.E. (1988) Predictors of Immediate and 6-Month Outcomes in Hospitalized Elderly Patients. The Importance of Functional Status. Journal of the American Geriatrics Society, 36, 775-783. https://doi.org/10.1111/j.1532-5415.1988.tb04259.x

[14] Inouye, S.K., Peduzzi, P.N., Robison, J.T., Hughes, J.S., Horwitz, R.I. and Concato, J. (1998) Importance of Functional Measures in Predicting Mortality among Older Hospitalized Patients. Journal of American Medical Association, 279, 1187-1193. https://doi.org/10.1001/jama.279.15.1187

[15] Purser, J.L., Kuchibhatla, M.N., Fillenbaum, G.G., Harding, T., Peterson, E.D. and Alexander, K.P. (2006) Identifying Frailty in Hospitalized Older Adults with Significant Coronary Artery Disease. Journal of the American Geriatrics Society, 54, 1674-1681. https://doi.org/10.1111/j.1532-5415.2006.00914.x

[16] Granton, J. and Cheng, D. (2008) Risk Stratification Models for Cardiac Surgery. Seminars in Cardiothoracic and Vascular Anesthesia, 12, 167-174. https://doi.org/10.1177/1089253208323681

[17] Geissler, H.J., Hölzl, P., Marohl, S., Kuhn-Régnier, F., Mehlhorn, U., Südkamp, M. and de Vivie, E.R. (2000) Risk Stratification in Heart Surgery: Comparison of Six Score Systems. European Journal of Cardio-Thoracic Surgery, 17, 400-406. https://doi.org/10.1016/S1010-7940(00)00385-7

[18] Society of Thoracic Surgeons (2015) Using Gait Speed to Measure Frailty. https://www.sts.org/sites/default/files/documents/ACSD_SpecUpgradeChangeSum maryV2_81.pdf

[19] Wilson, C.M., Kostsuca, S.R. and Boura, J.A. (2013) Utilization of a 5-Meter Walk Test in Evaluating Self-Selected Gait Speed during Preoperative Screening of Patients Scheduled for Cardiac Surgery. Cardiopulmonary Physical Therapy Journal, 24, 36-43.

[20] Enright, P.L. (2003) The Six Minute Walk Test. Respiratory Care, 48, 783-785.

[21] LaPier, T.K. and Mizner, R. (2009) Outcome Measures in Cardiopulmonary Physical Therapy: Focus on the Late Life Function and Disability Instrument (LLFDI). Cardiopulmonary Physical Therapy Journal, 20, 32-35.

[22] Jette, A.M., Haley, S.M. and Kooyoomjian, J.T. (2002) Late Life Function and Disa- 
bility Instrument. Trustees of Boston University, Boston, MA.

[23] Jette, A.M., Haley, S.M., Coster, W.J., Kooyoomjian, J.T., Levenson, S., Heeren, T. and Ashba, J. (2002) Late Life Function and Disability Instrument: I. Development and Evaluation of the Disability Component. The Journals of Gerontology: Series A, 57, M209-M216. https://doi.org/10.1093/gerona/57.4.M209

[24] Haley, S.M., Jette, A.M., Coster, W.J., Kooyoomjian, J.T., Levenson, S., Heeren, T. and Ashba, J. (2002) Late Life Function and Disability Instrument: II. Development and Evaluation of the Function Component. The Journals of Gerontology. Series A, 57, M217-M222. https://doi.org/10.1093/gerona/57.4.M217

[25] LaPier, T.K. and Waitt, M. (2007) Using the Late Life Function and Disability Instrument as a Self-Report Outcome Measure in Patients with Cardiovascular Disease. Journal of Cardiopulmonary Rehabilitation and Prevention, 27, 331. https://doi.org/10.1097/01.HCR.0000291340.61795.48

[26] LaPier, T.K. (2012) Utility of the Late Life Function and Disability Instrument as an Outcome Measure in Patients Participating in Outpatient Cardiac Rehabilitation: A Preliminary Study. Physiotherapy Canada, 64, 53-62. https://doi.org/10.3138/ptc.2010-30

[27] Sayers, S.P., Jette, A.M., Haley, S.M., Heeren, T.C., Guralnik, J.M. and Fielding, R.A. (2004) Validation of the Late-Life Function and Disability Instrument. Journal of the American Geriatrics Society, 52, 1554-1559.

https://doi.org/10.1111/j.1532-5415.2004.52422.x

[28] Denkinger, M.D., Igl, W., Coll-Planas, L., Bleicher, J., Nikolaus, T. and Jamour, M. (2009) Evaluation of the Short Form of the Late-Life Function and Disability Instrument in Geriatric Inpatients: Validity, Responsiveness, and Sensitivity to Change. Journal of the American Geriatrics Society, 57, 309-314. https://doi.org/10.1111/j.1532-5415.2008.02095.x

[29] Dubuc, N., Haley, S.M., Ni, P., Kooyoomjian, J.T. and Jette, A.M. (2004) Function and Disability in Late Life: Comparison of the Late-Life Function and Disability Instrument to the Short-Form-36 and the London Handicap Scale. Disability and Rehabilitation, 26, 362-370. https://doi.org/10.1080/09638280410001658667

[30] Beauchamp, M.K., Schmidt, C.T., Pedersen, M.M., Bean, J.F. and Jette, A.M. (2014) Psychometric Properties of the Late-Life Function and Disability Instrument: A Systematic Review. BMC Geriatrics, 29, 12.

[31] Bennett, J.A., Riegel, B., Bittner, V. and Nichols, J. (2002) Validity and Reliability of the NYHA Classes Measuring Research Outcomes in Patients with Cardiac Disease. Heart \& Lung, 31, 262-270. https://doi.org/10.1067/mhl.2002.124554

[32] LaPier, T.K. and Howell, D. (2002) Functional Status Deficits in Patients Recovering from Coronary Artery Bypass: An Application of Evidence-Based Practice. Cardiopulmonary Physical Therapy Journal, 13, 12-19. 